\title{
Interpretive Therapy
}

National Cancer Institute

\section{Source}

National Cancer Institute. Interpretive Therapy. NCI Thesaurus. Code C21056.

Psychotherapy of the Freudian school. In this form of psychotherapy, symbols from the patient's verbalizations, dreams, and interactions are interpreted for meaning in their everyday lives. Some mental health professionals find this very helpful in defining effective methods for treating chronic pain and pain syndromes. 